\title{
Alterstice
}

Revue internationale de la recherche interculturelle

International Journal of Intercultural Research

Revista International de la Investigacion Intercultural

\section{Inclusion, diversité et approche personnalisée : l'articulation d'un « nouveau » cadre éducatif en Colombie-Britannique}

\section{Marianne Jacquet}

Volume 6, numéro 1, 2016

Prendre en compte la diversité à l'école

URI : https://id.erudit.org/iderudit/1038286ar

DOI : https://doi.org/10.7202/1038286ar

Aller au sommaire du numéro

Éditeur(s)

Alterstice

ISSN

1923-919X (numérique)

Découvrir la revue

Citer cet article

Jacquet, M. (2016). Inclusion, diversité et approche personnalisée :

l'articulation d'un " nouveau " cadre éducatif en Colombie-Britannique.

Alterstice, 6(1), 147-158. https://doi.org/10.7202/1038286ar
Résumé de l'article

L'éducation inclusive représente « la voie de l'avenir " selon le Conseil des ministres de l'Éducation du Canada, et plusieurs initiatives à cet effet ont été mises en oeuvre dans différentes provinces canadiennes. En Colombie-Britannique (C.-B.), la démarche inclusive se déploie dans les politiques éducatives de reconnaissance de la diversité (langue, culture, " race ", religion, genre, etc.) et s'inscrit également en creux des principes d'égalité, de respect des droits, de dialogue interculturel et de justice sociale. Ces principes se déclinent dans l'ensemble des programmes scolaires, du primaire et du secondaire, ainsi que dans les objectifs de formation des enseignants au niveau universitaire. Plus récemment, le ministère de l'Éducation de la C.-B. s'est engagé dans une refonte majeure de son système éducatif afin de répondre aux exigences d'un monde en changement et aux besoins des apprenants du $21^{\mathrm{e}}$ siècle. Quelle forme prend alors la dyade " inclusion-diversité » dans la réforme des programmes en cours dans cette province ? Sur quels principes s'appuie-t-elle ? Nous traiterons dans cet article du Plan éducatif récent et de la manière dont l'inclusion de la diversité ethnoculturelle se décline dans ces nouveaux programmes scolaires.
Ce document est protégé par la loi sur le droit d'auteur. L'utilisation des services d'Érudit (y compris la reproduction) est assujettie à sa politique d'utilisation que vous pouvez consulter en ligne.

https://apropos.erudit.org/fr/usagers/politique-dutilisation/ 


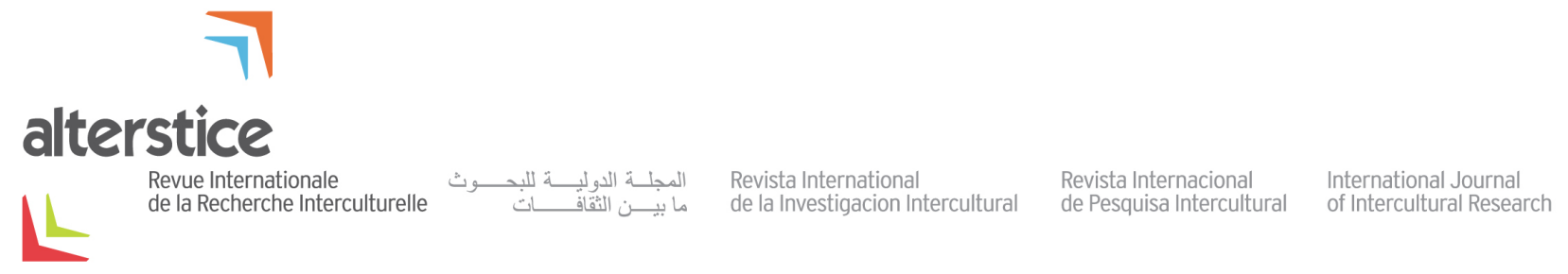

ARTICLE THÉMATIQUE

\section{Inclusion, diversité et approche personnalisée : l'articulation d'un "nouveau » cadre éducatif en Colombie-Britannique}

Marianne Jacquet ${ }^{1}$

\section{Résumé}

L'éducation inclusive représente " la voie de l'avenir » selon le Conseil des ministres de l'Éducation du Canada, et plusieurs initiatives à cet effet ont été mises en œuvre dans différentes provinces canadiennes. En ColombieBritannique (C.-B.), la démarche inclusive se déploie dans les politiques éducatives de reconnaissance de la diversité (langue, culture, "race ", religion, genre, etc.) et s'inscrit également en creux des principes d'égalité, de respect des droits, de dialogue interculturel et de justice sociale. Ces principes se déclinent dans l'ensemble des programmes scolaires, du primaire et du secondaire, ainsi que dans les objectifs de formation des enseignants au niveau universitaire. Plus récemment, le ministère de l'Éducation de la C.-B. s'est engagé dans une refonte majeure de son système éducatif afin de répondre aux exigences d'un monde en changement et aux besoins des apprenants du $21^{\mathrm{e}}$ siècle. Quelle forme prend alors la dyade " inclusion-diversité " dans la réforme des programmes en cours dans cette province ? Sur quels principes s'appuie-t-elle ? Nous traiterons dans cet article du Plan éducatif récent et de la manière dont l'inclusion de la diversité ethnoculturelle se décline dans ces nouveaux programmes scolaires.

\section{Rattachement de l'auteure}

${ }^{1}$ Simon Fraser University, Vancouver, Canada

\section{Correspondance}

mjacquet@sfu.ca

\section{Mots clés}

compétences interculturelles ; éducation ; politiques éducatives.

\section{Pour citer cet article}

Jacquet, M. (2016). Inclusion, diversité et approche personnalisée : I'articulation d'un « nouveau » cadre éducatif en Colombie-Britannique. Alterstice, 6(1), 147-158. 


\section{Introduction}

Dans le monde de l'éducation au Canada, comme ailleurs dans le monde, les notions "d'inclusion » et de " diversité » s'inscrivent en creux des politiques éducatives et des programmes scolaires (Conseil des ministres de l'Éducation du Canada [CMEC], 2008; Unesco, 2009) et reposent sur l'exigence du "vivre ensemble ». Souvent apposés dos à dos, l'inclusion et la diversité se caractérisent par leur multiplicité sémantique, laquelle à la fois enrichit le contenu des programmes scolaires et complexifie la démarche didactique.

L'éducation inclusive est présentée comme " la voie de l'avenir » selon le CMEC (2008) et les politiques éducatives dans différentes provinces canadiennes, comme la Colombie-Britannique (C.-B.), le Québec ou l'Ontario (Ministère de l'Éducation de la Colombie-Britannique [MECB], 2005; Ministère de l'Éducation de l'Ontario [MEO], 2009; Ministère de l'Éducation du Québec [MEQ], 2001) reflètent bien cette priorité. En C.-B. plus particulièrement, la démarche inclusive se déploie dans les politiques éducatives de reconnaissance de la diversité au sens large et s'arrime aux principes d'égalité, de respect des droits, de dialogue interculturel et de justice sociale (BC Multicultural Advisory Council, 2005; Jacquet, 2009; MECB, 2001 et 2005).

Jusqu'à présent, les thématiques relatives à la diversité ethnoculturelle (études autochtones, éducation au multiculturalisme et à l'antiracisme, etc.) et les principes énoncés dans les politiques éducatives étaient reproduits dans l'Annexe $C$ des programmes scolaires des niveaux d'enseignement primaire et secondaire. Or avec la refonte majeure du système éducatif de la province, lancée en 2011 pour répondre aux besoins des apprenants du $21^{\mathrm{e}}$ siècle et aux exigences d'un monde complexe et en changement, qui entraîne le déploiement progressif de nouveaux programmes scolaires, l'Annexe C n'existe plus. Comment la dyade "inclusion-diversité » est-elle alors mise en œuvre dans la réforme des programmes en cours dans la province ? Sur quels principes s'appuie-t-elle? Dans cet article, nous nous focalisons sur l'inclusion de la diversité ethnoculturelle dans le nouveau programme.

Dans un premier temps, nous présentons quelques repères discursifs sur la manière dont les textes officiels en éducation au Canada, et en C.-B. en particulier, traitent de la notion d'inclusion et de ses liens avec la notion de diversité. Ensuite, nous discutons plus en détail des principes qui structurent ce " nouveau » programme scolaire en C.-B. à travers l'analyse du Plan éducatif. Nous verrons notamment que le développement de trois compétences essentielles y occupe une place importante et que le contenu de l'Annexe C a laissé place à un traitement plus diffus et transversal de la problématique de la diversité ethnoculturelle.

\section{Diversité et inclusion : repères discursifs dans les politiques officielles de la Colombie-Britannique}

L'analyse qualitative du contenu des politiques éducatives officielles permet de faire émerger les thématiques importantes, les positionnements et les arguments avancés par l'institution éducative pour les justifier (Mucchielli, 2006). Elle donne à voir le(s) sens donnés aux notions de diversité et d'inclusion ainsi que leur articulation. Dans cet article, nous traitons du contenu explicite des discours, tel qu'il se déploie dans les documents officiels (politiques éducatives sur la diversité, Rapport Sullivan, Plan éducatif) et dans le programme scolaire. Pour l'analyse, nous avons retenu les sections explicitant les concepts utilisés ainsi que les positionnements officiels, en particulier dans le programme scolaire où seule la partie décrivant la nouvelle structure de programmes est retenue. Les extraits sélectionnés varient d'un à plusieurs paragraphes, selon les documents.

\section{Le discours inclusif : de l'inclusion des élèves à besoins spéciaux à l'inclusion des élèves issus des minorités}

L'utilisation du terme "inclusion » en éducation provient à la fois de la lutte pour les droits civils aux États-Unis (Bélanger et Duchesne, 2010) et de l'intégration des élèves à besoins spéciaux ou en situation de handicap dans les classes ordinaires et aux aménagements mis en œuvre pour faciliter leur apprentissage (Unesco, 2009). Toutefois, cette notion prend progressivement un sens plus global pour s'appliquer également aux élèves issus des minorités (culturelles, linguistiques, raciales et religieuses) marginalisées (Jacquet, 2014). 
Désormais, l'éducation inclusive est entendue :

[...] comme un processus qui vise à prendre en compte et à satisfaire la diversité des besoins des élèves de tous - enfants et adultes - par une participation accrue à l'apprentissage, à la vie culturelle et à la vie communautaire, et par une réduction du nombre de ceux qui sont exclus de l'éducation ou au sein même de l'éducation. Elle suppose de changer et d'adapter les contenus et les approches, les structures et les stratégies, en s'appuyant sur une vision commune qui englobe tous les enfants du groupe d'âge visés et avec la conviction que le système éducation ordinaire a le devoir d'éduquer tous les enfants. (Unesco, 2009, p. 9)

De son côté, le Conseil des ministres de l'Éducation du Canada ${ }^{1}$ (CMEC, 2008) définit « l'éducation pour l'inclusion comme l'exploration des moyens de transformer les systèmes éducatifs et autres contextes d'apprentissage pour tenir compte de la diversité des personnes qui apprennent. La qualité d'un système se mesure donc à la façon dont il encourage la pleine participation » (p. 38).

En C.-B. plus spécifiquement, le discours inclusif émerge à la fin des années 1980 dans un rapport de la Commission royale du Canada sur l'éducation connu sous le nom de Rapport Sullivan, du nom de l'auteur ayant mené ce vaste chantier de consultations auprès de divers acteurs scolaires (Sullivan, 1988). Dans ce volumineux rapport, le discours inclusif est défini en filigrane en référence à trois niveaux complémentaires : individuel, identitaire et géographique. Le premier niveau fait référence à la nécessité de reconnaître la diversité des besoins, des expériences et des modes d'apprentissage des élèves; le deuxième fait appel à la nécessité de préserver les héritages culturels et les préférences religieuses ou langagières et le troisième, plus global, insiste sur les différences identitaires des communautés et des régions, lesquelles conduisent l'école à devoir s'adapter à l'environnement spécifique du milieu desservi.

Throughout the study, individuals spoke and wrote about the need for school programs and operations to recognize the diversity that exists in British Columbia society. Public emphasis on diversity was many sided. To some, it was a question of making greater provision for individual differences among learners, addressing such issues as pupil streaming, differential programs in elementary schools, and the general challenge of furnishing young people with school experiences to match their interests and abilities. To others, it was an issue intertwined with the preservation of cultural heritage, religious preference, or language. Diversity also had a geographic meaning. In some instances, when people spoke of diversity they dealt with the importance of recognizing the distinct identities of regions, communities, or even neighbourhoods, and the need to allow local schools to reflect such differences in their offerings. (Sullivan, 1988, p. 6)

L'intérêt de cet extrait est qu'il préfigure les principes fondateurs qui articuleront près de deux décennies plus tard la politique de reconnaissance de la diversité ethnoculturelle dans l'espace scolaire en C.-B. autour des pôles inclusion-identités-communautés.

\section{Le discours sur la diversité : de la reconnaissance de la complexité}

Deux documents officiels du MECB tracent la voie de la reconnaissance de la diversité dans le système scolaire : Save, caring and orderly school. A guide (MECB, 2004) et La diversité dans les écoles de la C.-B. : document-cadre (MECB, 2005). Le premier énonce une vision de la culture scolaire propre à favoriser l'inclusion. Le second traite de l'étendue et des défis de l'inclusion de la diversité dans les écoles en ancrant la discussion sur les concepts de justice sociale, d'équité, de droits de la personne et de multiculturalisme. Il suggère également aux commissions scolaires des pistes permettant de mettre en œuvre des initiatives inclusives dans les écoles. Nous rejoignons ici une conception de l'inclusion étroitement associée à la reconnaissance des droits fondamentaux de la personne et aux facteurs contextuels limitant la pleine participation des personnes à l'école en toute égalité (Bélanger et Duchesne, 2010).

Dans son cadre de référence sur la diversité, le MECB introduit la complexité dans son approche conceptuelle de la diversité en éducation. Celle-ci est en effet définie comme un « concept global » qui inclut la référence à plusieurs marqueurs sociaux : I'origine ethnique, la langue, la culture, les croyances religieuses, la " race ", le genre, l'âge, le milieu socioéconomique et la diversité des habiletés cognitives et physiques des apprenants.

\footnotetext{
${ }^{1}$ Au Canada, les systèmes éducatifs sont de juridiction provinciale.
} 
La diversité se rapporte aux particularités qui nous différencient les uns des autres. Certaines différences sont visibles (p. ex. race, origine ethnique, sexe, âge, aptitudes), tandis que d'autres le sont moins (culture, ascendance, langue parlée, croyances religieuses, orientation sexuelle, milieu socioéconomique). (MECB, 2005, p. 7)

En corollaire, cette politique retient une conception fluide de l'identité des élèves située dans le jeu du rapport à soi (individualité) et à celui de sa ou ses communauté(s) d'appartenance :

[C]e concept [de diversité] englobe à la fois l'idée de notre individualité et notre sentiment d'appartenance ou notre besoin d'identification à un ou à des groupes. (p. 7)

Dès lors, la posture de reconnaissance des identités et du lien communautaire s'inscrit au cœur du rapport de l'école à la diversité ethnoculturelle. Ce positionnement fait en cela écho à celui défendu par Taylor (1994) dans Multiculturalisme, différence et démocratie, ouvrage dans lequel il s'appuie sur les idéaux modernes d'authenticité et d'autonomie pour défendre l'idée de permettre à un individu de développer sa propre identité car, selon l'auteur, une image négative ou dévalorisante des identités de certaines communautés conduit à une forme d'aliénation et de domination.

Résumant la recherche sur l'équité dans le traitement des groupes minorisés dans les institutions scolaires, Potvin et Larochelle-Audet (2016) avancent que la prise en compte de la problématique ethnoculturelle à l'école s'inscrit dans le prolongement des travaux réalisés par les théoriciens critiques et antiracistes, lesquels ont souligné l'importance pour les acteurs scolaires de focaliser sur une compréhension fine des rapports de pouvoirs entre les groupes majoritaires et minoritaires, ainsi que sur la complexité des processus d'oppression et des multiples facteurs (identitaires, sociaux, structurels, historiques, linguistiques et économiques) interagissant mutuellement pour générer des situations discriminatoires au sein des institutions scolaires.

Les politiques d'éducation interculturelle et multiculturelle, déployées dans les années 1980 au Canada comme ailleurs dans le monde (Abdallah-Pretceille, 2003; Belkaïd, 2002 ; Kanouté, 2007 ; Lorcerie, 2002 ; Moodley, 1988; Potvin, Magnan et Larochelle-Audet, 2016), émergent en réponse à la diversité ethnoculturelle dans les écoles et au besoin de développer une image positive des langues et des identités culturelles des élèves afin d'appuyer leurs apprentissages scolaires.

S'il ne faut pas négliger les critiques faites à l'égard de ces approches en raison d'une représentation folklorisante des cultures et d'un manque d'attention portée aux phénomènes de racisme et de discrimination systémique dans les institutions éducatives (Dei, 1999 ; Gérin-Lajoie et Jacquet, 2008 ; Ladson-Billings, 2004 ; Potvin et Carr, 2008), ces approches ont néanmoins permis aux acteurs scolaires d'envisager les différences ethnoculturelles non plus en termes de "déficit» (Moodley, 1988) mais comme des atouts pour améliorer les conditions dans lesquels l'apprentissage se produit. En cela, elles posent les fondements théoriques et didactiques d'une conception inclusive de la diversité à visée transformative, et où les expériences et les savoirs des apprenants sont considérés comme des atouts et des leviers d'apprentissage (Litalien, Moore et Sabatier, 2012). Ces principes sont clairement exprimés dans le cadre de référence du MECB, La diversité dans les écoles de la C.-B. :

Le respect de la diversité repose sur le principe voulant que la reconnaissance et l'utilisation constructive des différences aient pour effet d'améliorer la qualité de nos milieux d'apprentissage et de travail. (MECB, 2005, p. 7)

Plus largement, en C.-B., le discours sur l'inclusion de la diversité s'inscrit dans un contexte social favorable à la reconnaissance de la diversité ethnoculturelle, comme le Multiculturalism Act (Gouvernement de la ColombieBritannique, 1996) et dans la foulée des approches multiculturelle et de l'antiracisme adoptées dans la province. L'inclusion de la diversité en éducation s'inscrit également dans une conception citoyenne du "vivre ensemble " dans une société multiethnique, et repose sur des principes d'égalité, de respect des droits, de justice sociale et de dialogue interculturel (MECB, 2001, 2005 et 2008). Pour le MECB, la recherche de l'équité par le biais de "l'inclusion sociale" concerne tout autant les milieux scolaires que la société dans son ensemble. L'approche inclusive permet ainsi d'intégrer les principes clés des approches éducatives multiculturelle, de l'antiracisme et de la citoyenneté (Potvin et Larochelle, 2016). 
La conception de l'inclusion de la diversité retenue par le MECB est à la fois riche et complexe. Sa richesse tient à ce que la posture inclusive s'inscrit dans une visée transformative. Elle s'appuie sur une écologie de la diversité (Litalien, Moore et Sabatier, 2012) permettant de consolider le lien social entre l'école et le milieu culturel desservi, de favoriser la reconnaissance de l'identité singulière et plurielle des élèves, de valoriser le partenariat avec les familles et enfin d'engager l'ensemble des acteurs scolaires à la mise en œuvre de l'équité et de la justice sociale à tous les niveaux de l'organisation scolaire.

Sa complexité tient à ce que l'inclusion de la diversité pose inévitablement des défis au niveau de sa modélisation dans les pratiques scolaires, en particulier au niveau du programme, lorsqu'il s'agit d'équilibrer la pluralité des sensibilités morales des communautés ethnoculturelles desservies à l'école et les exigences du "vivre ensemble " (Jacquet et D'Amico, 2016). En C.-B., ces défis ont généré des tensions importantes, ce qu'illustre un jugement de la Cour Suprême du Canada (Chamberlain v. Surrey School District No. 36) ${ }^{2}$. À cette complexité inhérente s'ajoute la méconnaissance par les acteurs scolaires des politiques éducatives sur la diversité et le manque de formation à ce sujet (Gérin-Lajoie et Jacquet, 2008).

En somme, l'approche inclusive en éducation portée par les discours institutionnels comporte une zone d'ombre autour des modalités concrètes de la reconnaissance dans les pratiques scolaires. Si le Rapport Sullivan (1988) faisait explicitement référence à certaines contraintes ${ }^{3}$, aujourd'hui, rien de tel ne transpire dans le discours éducatif circulant autour de l'inclusion : le sujet est tout simplement évacué. Pourtant, près de trois décennies plus tard, l'enjeu est loin d'être clos et les enseignants et administrateurs ne sont pas mieux formés pour négocier les tensions et les contradictions axiologiques que génèrent à l'occasion les demandes de reconnaissance des identités culturelles et religieuse (Bourgeault, Gagnon, Mc Andrew et Pagé, 1995; Clarke, 2005 ; Jacquet et D’Amico, 2016 ; Shariff, 2006). De plus, il n'est pas certain non plus que les nouvelles orientations du Plan éducatif permettent d'y répondre.

Finalement, ce que dévoilent ces discours, c'est bien la nature instable de l'inclusion, conçue comme un idéal à atteindre, un horizon, plutôt que comme un processus négocié par les acteurs scolaires (Bélanger et Duchesne, 2010).

\section{Les principes fondateurs du nouveau programme scolaire en Colombie-Britannique}

\section{Le "nouveau » Plan d'éducation de Colombie-Britannique}

En 2010, le MECB s'est engagé dans un vaste processus de consultation auprès de ses différents partenaires (enseignants, élèves, parents et autres Britanno-Colombiens) afin de revoir son Plan éducatif. Lancé en octobre 2011, le «nouveau » Plan éducatif (MECB, 2015b, p. 3) propose une refonte majeure des programmes scolaires (maternelle à $12^{\mathrm{e}}$ année) permettant d'articuler plus étroitement les besoins des élèves aux exigences d'un monde en changement. Dans le prolongement de la vision citoyenne et solidaire élaborée dans le Cadre de référence sur la diversité (Litalien, Moore et Sabatier, 2012), le Plan éducatif s'appuie sur le développement de compétences essentielles respectueuses des contextes culturels dans lesquels se déroule l'acte pédagogique.

Les orientations générales de ce plan sont de favoriser une approche plus personnalisée de l'apprentissage en donnant aux élèves une plus grande liberté pour aborder des sujets et des activités qui les passionnent, d’impliquer plus étroitement les parents à la planification de l'éducation de leurs enfants pour les aider à réussir, d'intervenir plus efficacement auprès des élèves en difficulté et enfin de poursuivre les ententes de partenariat avec les

\footnotetext{
${ }^{2}$ Ce jugement porte sur le refus d'une commission scolaire d'autoriser un enseignant d'une classe de première année du primaire d'utiliser avec ses élèves trois livres illustrant des familles de même sexe (One Dad, Two Dads, Brown Dad, Blue Dads, Asha's Mum et Belinda's Bouquet) afin de sensibiliser ses élèves à la diversité des types de famille, ce qui est recommandé dans les objectifs du programme scolaire de la province. Pour une description complète de ce jugement, consulter: http://www.canlii.org/fr/ca/csc/doc/2002/2002csc86/2002csc86.html\#showHeadnotes.

3 "Today, we look to the schools to help us sustain a climate in which racial, religious, and linguistic differences will be both understood and appreciated, even if such differences are not easily accommodated within the framework of any one system " (p. 10-11).
} 
communautés autochtones, par l'entremise des districts scolaires, afin d'améliorer la performance scolaire des élèves autochtones, dont le taux de diplomation est le plus faible au Canada. En effet, selon Statistique Canada (2011), 48,5 \% des membres des Premières Nations n'ont aucun certificat, diplôme ou grade, contre $12,1 \%$ des populations non autochtones.

La transition vers le nouveau programme est progressive : elle a commencé en 2015 en fonction des niveaux d'enseignement et va se poursuivre jusqu'en 2018. Par exemple, à la rentrée 2015, les enseignants pouvaient utiliser sur une base optionnelle les nouveaux programmes de la maternelle à la $9^{e}$ année. À la rentrée scolaire 2016, ces derniers sont dorénavant obligatoires. D'autres programmes (niveaux 10 à 12) sont encore à l'état d'ébauche, de même que la révision des critères d'évaluation et l'arrimage entre le nouveau programme et les exigences de diplomation à la fin du secondaire.

S'il est encore trop tôt pour analyser la pratique de l'inclusion de la diversité ethnoculturelle dans les classes alors que ce changement est en cours, il est utile de rappeler que la centration sur l'élève s'inscrit dans la continuité des travaux en éducation. En effet, Hansman (2016) rappelle que la centration sur l'apprenant n'est pas nouvelle car Dewey, dans Democracy and Education (1916), argumentait déjà sur l'importance de placer l'élève au cœur de l'apprentissage plutôt que le programme au centre de la classe. Elle est également abordée sous différentes formes dans le Rapport Sullivan (1988), comme l'indiquent les trois extraits suivants :

The Government of British Columbia believes that education is a lifelong process embracing many facets, including personal development, career preparation, the enhancement of creativity, self-discipline, mature judgement, and a broad range of life skills, not the least of which is curiosity-the love of learning. (p. 1)

Many [parents] who appeared before the Commission argued for greater choice and variety in schooling as a means of recognizing individual differences among youngsters, as well as acknowledging parental rights to select the academic, social, and moral atmosphere of schools in accordance with their own philosophical beliefs and values. (p. 7)

To some, it was a question of making greater provision for individual differences among learners, addressing such issues as pupil streaming, differential programs in elementary schools, and the general challenge of furnishing young people with school experiences to match their interests and abilities [...]. (p. 7)

Dès lors, la «nouveauté " tient à la prise en compte des connaissances et des perspectives autochtones ${ }^{4}$ dans l'ensemble des programmes et à l'ancrage pédagogique autour de la notion de "compétences transversales » ${ }^{5}$.

\section{Les compétences essentielles transversales}

Le nouveau programme de la C.-B. s'appuie sur une approche par compétences permettant d'articuler savoirs, savoir-faire, savoir-comprendre et savoir-être. L'approche par compétences fait partie du vocabulaire courant de divers champs de formation (professionnelle et technique, collégiale, universitaire) et s'inscrit en creux des discours sur la professionnalisation des enseignants (Altet, Paquay et Perrenoud, 2002; Legendre et Morrissette, 2014; Perrenoud, 2004; Potvin, Magnan et Larochelle-Audet, 2016). Cette perspective implique également une responsabilité de la part des enseignants à rendre compte de leurs actions pédagogiques sur les résultats attendus. Pour Duru-Bellat (2007), dans la mesure où l'objectif poursuivi est l'équité et si l'enfant est situé au cœur de la démarche pédagogique, il devient nécessaire " $\left[d^{\prime}\right]$ évaluer la manière optimale d'organiser le contexte scolaire pour maximiser les résultats de l'action pédagogique sur les résultats de l'élève " (cité dans Legendre et Morrissette, 2014, p. 214).

\footnotetext{
${ }^{4}$ Nous ne traiterons pas de cette dimension dans cet article car elle mérite à elle seule un traitement indépendant et plus approfondi.

${ }^{5}$ Ces compétences furent énoncées dans une politique précédente (en 2000), laquelle ne fut jamais mise en œuvre suite au changement de gouvernement (Hansman, 2016).
} 
Cependant, l'approche par compétences ne fait pas l'unanimité : elle produit des discours très opposés. Pour les uns, elle marque l'assujettissement de l'école aux exigences du marché du travail alors que pour les autres elle favorise une plus grande démocratisation de l'école et une adaptation aux nouvelles réalités culturelles et économiques (Legendre et Morrissette, 2014). S'appuyant sur une discussion de la littérature, ces auteures avancent ainsi que :

Dans cet esprit, l'usage des savoirs auquel on associe l'idée de compétences ne se réduit pas à une conception utilitariste étroite, mais revêt un sens beaucoup plus large de mobilisation des ressources, tant externes qu'internes, témoignant de leur intégration et de leur appropriation. Cette mobilisation, qui va bien au-delà de la restitution de connaissances mémorisées, exige à la fois le recours à des situations d'apprentissage d'un certain niveau de complexité, et d'un soutien important de l'enseignant (Legendre et Morrissette, 2014, p. 231).

Pour le MECB (2015), la notion de compétence renvoie à :

[...] La capacité des élèves à accomplir de manière adéquate une tâche liée à une discipline ou à un domaine d'apprentissage précis. Cette capacité englobe des habiletés, des processus, des comportements et des modes de pensée. Les élèves sont compétents dans un domaine d'apprentissage dans la mesure où ils comprennent des connaissances et peuvent les mettre en pratique dans de nouveaux contextes. (p. 5)

Trois compétences essentielles sont discutées dans les nouveaux programmes, en plus des compétences de base en numératie et littératie : la compétence de pensée $(P)$, la compétence de communication $(C)$ et les compétences personnelles et sociales (PS). Ces compétences sont transversales à l'ensemble des programmes des niveaux d'enseignement primaire et secondaire : elles visent à former des citoyens informés et à favoriser l'apprentissage continu tout au long de la vie.

La compétence de pensée (CP) repose sur le développement de la pensée critique et créative. Il s'agit des habiletés cognitives que l'élève développe tout au long de ses études. La compétence à communiquer (CC) focalise sur les habiletés dont l'élève a besoin pour apprendre et interagir efficacement dans le monde. Enfin, les compétences personnelles et sociales (CPS) représentent les habilités sociales et culturelles que les élèves développent en tant qu'individus et membres de la société (MECB, 2013b, p. 3). Chacune de ces compétences se décline en différentes composantes auxquelles sont attachées des habiletés spécifiques.

Ces compétences transversales peuvent être qualifiées de "haut niveau " et d'holistiques, puisqu'elles mobilisent une combinaison de savoirs, savoir-comprendre, savoir-faire et savoir-être imbriqués les uns aux autres, qui visent à développer les habiletés cognitives, communicatives ainsi que les habiletés personnelles et sociales des élèves nécessaires à la formation de citoyens éclairés (Legendre et Morrissette, 2014).

La notion de compétences interculturelles n'est pas mentionnée dans ce programme, mais elle se dévoile en filigrane dans les trois composantes des compétences PS : une identité personnelle et culturelle positive, une prise de conscience de ses responsabilités personnelles et une prise de conscience de ses responsabilités sociales.

A positive personal and cultural identity is the awareness, understanding and appreciation of one's own ancestry, culture, language, belief and perspective in a multi-cultural society. [...]. Personal awareness and responsibility is the ability to understand and take responsibility for one's actions, including one's learning, to make constructive and ethical decisions about one's personal and social behaviour, and to accept consequences and understand how one's actions affect one's own well-being and that of others [...]. Social awareness and responsibility is the ability to cooperate and collaborate with others, empathize with and appreciate the perspective of others, and create and maintain healthy relationships within one's family, community, and society. (MECB, 2013b, p. 7)

En effet, chacune de ces composantes mobilise des savoir-faire et des savoir-être complexes (Potvin, Magnan et Larochelle-Audet, 2016) qui font écho aux composantes des compétences interculturelles discutées dans la littérature nord-américaine et européenne sur la formation des enseignants à la diversité ethnoculturelle (BorriAnadon, Larochelle-Audet, Potvin et McAndrew, 2014; Lázár, 2005 ; Potvin, Magnan et Larochelle-Audet, 2016), comme celle sur l'enseignement des langues (Byram, 2011 ; Byram, Gribkova et Starkey, 2002 ; Conseil de l'Europe, 2001 ; Jacquet et Côté, 2014 ; Lussier, 2007 ; Ragoonaden, 2011 et 2012). 
Le savoir-être est d'ordre affectif et cognitif, il a trait aux représentations mentales que l'élève développe sur le monde qui l'entoure ainsi que sur les attitudes d'ouverture ou de rejet à l'égard d'autres cultures. Selon Ragoonaden (2012), le savoir-être se décline en trois stades, allant de la sensibilité culturelle, en passant par une meilleure connaissance de soi, de sa culture et de son identité (centration) et en se poursuivant par un processus de décentration permettant de mettre en évidence les compétences interculturelles au travers de " l'internalisation de ses propres valeurs, la valorisation des valeurs des autres cultures et la capacité de médiation culturelle » (p. 37).

Ces trois stades du savoir-être se déclinent dans les trois habiletés spécifiques associées à la composante identitaire, lesquelles portent d'abord sur l'influence des relations et des contextes culturels dans la construction identitaire ("les élèves comprennent que leurs relations et leurs contextes culturels contribuent à forger leur identité [...] », MECB, n.d.), puis sur l'orientation des valeurs (" les élèves définissent leurs valeurs. Ils comprennent que ces valeurs ont été influencées par leurs expériences de vie et comment les valeurs influencent les choix qu'ils font dans toutes les sphères de leur vie. " $M E C B$, n.d.) et enfin sur l'identification et le réinvestissement des forces et aptitudes personnelles de l'élève au sein de sa famille et de la communauté en général.

La composante responsabilité sociale comprend quant à elle quatre habiletés spécifiques, dont l'une cible la reconnaissance de la diversité : " les élèves valorisent la diversité, défendent les droits de la personne et les droits d'autrui, en plus de faire preuve d'un sens éthique dans leurs interactions, y compris celles en ligne » (MECB, n.d.).

Ces composantes ne sont pas nécessairement nouvelles puisqu'on les retrouve dans certains documents officiels et programmes scolaires: Responsabilité sociale: Document-cadre (MECB, 2001), Making Space: Teaching for diversity and social justice throughout the $K$-12 curriculum (MECB, 2008) et le Social justice 12 curriculum (MECB, 2008). Toutefois, elles sont dorénavant mieux intégrées dans l'ensemble des programmes.

Finalement, puisqu'elles s'inscrivent dans une écologie de la diversité (Litalien, Moore et Sabatier, 2012), les compétences de pensée, de communication et les compétences personnelles et sociales doivent être enseignées de manière à équilibrer les besoins individuels (la focalisation sur l'apprenant) et les besoins collectifs (les contextes culturels particuliers):

[...] it is necessary both to value the different contexts of students' lives and learning environments and to define competencies in a way that is open to different cultural and social interpretations. For example, in the context of Aboriginal learners, the cross-curricular competencies may be interpreted in a way that respects the mental, physical, spiritual, and emotional aspects of a whole and healthy being. Other cultural contexts may require similar or unique cultural interpretations in order to be meaningful within the context of students' whole lives. (MECB, 2013b, p. 4)

Cet énoncé présente l'articulation de la dyade "inclusion-diversité " au travers du développement des compétences transversales, dont la mise en œuvre dans l'agir pédagogique doit être flexible afin de tenir compte des environnements culturels du milieu. II ne s'agit pas simplement de prendre en compte la différence culturelle de manière superficielle, mais bien d'engager les élèves comme les enseignants dans un processus de décentration culturelle (Abdallah-Pretceille, 2003) et épistémologique permettant de comprendre l'Autre à travers la manière dont il construit ses propres modes de connaissance. Dès lors, le développement des compétences essentielles est modulable en fonction des contextes scolaires et de la sensibilité morale ou religieuse des communautés desservies.

Durant cette période transitoire, il n'est pas possible, à la seule lecture des documents officiels, de bien saisir toutes les nuances et les défis auxquels vont être confrontés les acteurs scolaires dans une mise en œuvre nuancée des compétences essentielles dans leurs divers contextes scolaires. Toutefois, lors d'un congrès récent organisé en avril 2016 par la BC Modern Language Association (BCLCA), des questions ont été soulevées par les participants, lors d'activités en groupe, sur la capacité à développer la pensée critique chez les élèves alors même que le ministère de l'Éducation semble retenir une conception rigide de la culture. 
En effet, la flexibilité avec laquelle il est possible de développer les compétences essentielles en fonction des contextes culturels ne doit pas faire oublier les variations intra-groupales. Dans un article récent, AbdallahPretceille (2011) soutient en effet que le multiculturalisme est souvent perçu comme une addition de groupes culturels. Or, selon l'auteure, il importe de tenir compte de la tension entre l'individuel et le collectif de sorte que « [l]e rattachement systématique d'un comportement particulier à une appartenance culturelle groupale doit être nuancé par rapport à des variations intra-groupales. » (p. 93). Pour l'auteure, les cultures sont avant tout des modes opératoires permettant de parler des cultures mais ne correspondant pas à une réalité. La manière d'équilibrer la tension entre le collectif et l'individuel est peu discutée dans ce nouveau programme, pas plus que la manière de tisser le lien social au-delà des identités culturelles ou religieuses particulières. L'interrogation est légitime, et une analyse plus fine des différents programmes et des pratiques sur le terrain reste à faire afin de bien saisir la mise en acte des compétences essentielles dans les contextes scolaires desservant différentes communautés ethnoculturelles.

\section{Conclusion}

Le nouveau programme s'appuie sur une approche d'enseignement personnalisée, adaptée aux besoins de l'éducation au $21^{\mathrm{e}}$ siècle, où les enseignants n'agissent plus en tant que source unique de connaissances, mais assument des rôles diversifiés: guide, mentor, entraîneur et spécialiste de contenu. Il est structuré autour de savoirs imbriqués les uns aux autres: les savoirs (contenus disciplinaires), les savoirs-faire (compétences essentielles et compétences disciplinaires), les savoirs-comprendre (les grandes idées) et les savoirs-être (les attitudes).

Dans ce nouveau programme, l'articulation de la dyade "inclusion-diversité » se manifeste de manière diffuse au travers du développement des compétences essentielles : elle s'intègre à une écologie de la diversité (Litalien, Moore et Sabatier, 2012) permettant de moduler le développement des compétences transversales en tenant compte de l'environnement culturel du milieu desservi. À cet égard, le nouveau programme fait une large place à l'intégration systématique des connaissances et aux perspectives autochtones afin de redresser les discriminations passées et de développer une compréhension et un respect mutuels. Cette dimension importante (qui n'a pas fait l'objet d'une discussion élaborée dans cet article en raison de contraintes d'espace) mériterait d'être explorée davantage.

En somme, ce nouveau programme met en acte un changement de paradigme reposant sur une approche personnalisée de l'enseignement afin de répondre à un monde globalisé et en changement, et dont on retrouve les traces dans le Rapport Sullivan (1988). Cette transformation des programmes est riche de possibilités pour les élèves, les familles et les communautés, en particulier celles historiquement marginalisées, puisqu'elle suggère de s'appuyer plus directement sur leurs savoirs, leurs expériences et leurs besoins dans la construction de l'acte pédagogique. Comme nous l'avons vu, il est encore difficile à la seule lecture de documents officiels de saisir tous les défis auxquels vont être confrontés les acteurs scolaires dans l'application nuancée des compétences essentielles en fonction des communautés ethnoculturelles desservies. À cet égard, une analyse plus approfondie du contenu des matières enseignées et de la transformation de l'agir pédagogique des enseignants reste à faire. Cette analyse permettra de mettre à jour la manière dont les tensions entre l'individuel et le collectif (AbdallahPretceille, 2011) se manifestent dans l'enseignement des matières, ainsi que la manière dont les interprétations en tension vont se négocier au niveau de la classe, de l'école ou du district scolaire.

\section{Références bibliographiques}

Abdallah-Pretceille, M. (2003). Former et éduquer en contexte hétérogène. Pour un humanisme du divers. Paris : Anthropos/ Economica.

Abdallah-Pretceille, M. (2011). La pédagogie interculturelle : entre multiculturalisme et universalisme. Lingvarum Arena, 2, 91-101.

Altet, M., Paquay, L. et Perrenoud, P. (2002). Formateurs d'enseignants : Quelle professionnalité ? Bruxelles : De Boeck. 
BC Multicultural Advisory Council (BCME) (2005). Strategic framework for action : a strategy to stimulate joint action on multiculturalism and the eliminatation of racism in Britsih Columbia. Submitted to the Honourable Murray Coell, Minister of Community, Aboriginal and Women's Services. www.bced.gov.bc.ca/reporting.

Bélanger, N. et Duchesne, H. (2010). Des écoles en mouvement. Inclusion des élèves en situation de handicap ou éprouvant des difficultés à l'école. Ottawa : Presses de l'université d'Ottawa.

Belkaïd, M. (2002). La diversité culturelle : pour une formation des enseignants en altérité. Raisons éducatives, 1, 205-222.

Borri-Anadon, C., Larochelle-Audet, J., Potvin, M. et McAndrew, M. (2014). Bilan et enjeux de la formation initiale à la diversité ethnoculturelle, religieuse et linguistique en enseignement au Québec. Diversité canadienne 11(2), 59-64.

Bourgeault, G., Gagnon, F., Mc Andrew, M. et Pagé, M. (1995). L'espace de la diversité culturelle et religieuse à l'école dans une démocratie de tradition libérale. Revue des migrations internationales, 11(3), 79-103.

Byram, M. (2011). La Compétence interculturelle. Dans P. Blanchet et P. Chardenet (dir.), Guide pour la recherche en didactique des langues et des cultures (p.253-260). Paris : Éditions des archives contemporaines.

Byram, B., Gribkova, H. et Starkey, S. (2002). Développer la dimension interculturelle dans l'enseignement des langues. Strasbourg : le Conseil de L'Europe.

Clarke, P. (2005). Religion, public education and the Charter: where do we go now? McGill Journal of Education, 40(3), 351-381.

Conseil de l'Europe (2001). Cadre européen commun de référence pour les langues : apprendre, enseigner, évaluer. Division des langues vivantes. Paris : Didier.

Conseil des ministres de l'Éducation du Canada (en coll. avec la Commission canadienne de l'Unesco) (2008). Second Rapport - L'éducation pour l'inclusion au Canada : la voie de l'avenir. http://www.ibe.unesco.org/National_Reports/ICE_2008/canada_NR08_fr.pdf

Cour suprême du Canada (2002). Chamberlain v. Surrey School District No. 36, [2002] 4 SCR 710, 2002 SCC 86. [traduction : https://scc-csc.lexum.com/scc-csc/scc-csc/fr/item/2030/index.do]

Dei, G. (1999). The denial of difference: Reframing antiracist praxis. Race, Ethnicity and Education, 2(1), 17-37.

Duru-Bellat, M. (2007). L'évaluation de la qualité du contexte scolaire : dérive managériale ou exigence démocratique ? Dans M. Behrens (dir.), La qualité en éducation pour réfléchir à la formation de demain (p. 127-155). Québec : Presses de l'Université du Québec.

Gérin-Lajoie, D. et Jacquet, M. (2008). Regards croisés sur l'inclusion des minorités en contexte scolaire francophone minoritaire au Canada. Numéro spécial « Rapports ethniques et éducation : perspectives nationales et internationales ", Revue éducation et francophonie, 36(1), 25-43.

Gouvernement de la Colombie-Britannique. Multiculturalism Act [RSBC 1996], chap. 321. Victoria : Gouvernement de la Colombie-Britannique. [Traduction :http://www.axl.cefan.ulaval.ca/amnord/colombiebritlois_divers.htm]

Hansman, G. (2016). Personalized learning Back to the Future? Teacher, 28(3), 6-7.

Jacquet, M. (2009). L'espace marginal de l'inclusion de la diversité ethnique à l'école : I'exemple de la ColombieBritannique. Canadian Ethnic Studies, 41(1-2), 95-113.

Jacquet, M. (2014). La formation interculturelle des enseignants en contexte francophone minoritaire : exemples de dispositifs pédagogiques. Dans Laurie Carlson-Berg (dir.), La francophonie dans toutes ses couleurs :

Discours sur l'inclusion scolaire en milieu francophone canadien (p. 99-115). Québec : Presses de l'Université Laval. 
Jacquet, M. et Côté, I. (2014). L'utilisation de la bande dessinée en salle de classe : un outil de formation interculturelle critique. Revue de l'Association québécoise des enseignants de français langue seconde, 31(1), 150-165.

Jacquet, M. et D’Amico, L. (2016). Religious identity and inclusion: Policy and accommodation practices in British Columbian secular schools system. Canadian Journal of Education, 39(2), 1-25.

Kanouté, F. (2007). La pratique de l'interculturel. Dans C. Solar et F. Kanouté (dir.), Questions d'équité en éducation et formation (p. 121-142). Montréal : Éditions Nouvelles.

Kruger-Potratz, M. (2003). Éducation et formation interculturelle en Allemagne. Dans M. Abdallah-Pretceille (dir.), Former et éduquer en contexte hétérogène. Pour un humanisme du divers (p. 155-172). Paris : Anthropos/Economica.

Ladson-Billings, G. (2004). Just what Is critical race theory and what's it doing in a nice field like education? Dans G. Ladson-Billings et D. Gillborn (dir.), The Routledge Falmer Reader in Multicultural Education (p. 49-67). London : Routledge Falmer Publishing Company.

Lázár, I. (2005). Intégrer la compétence interculturelle dans la formation des enseignants. Strasbourg : Conseil de l'Europe.

Legendre, M.-F. et Morrissette, J. (2014). Défis et enjeux de l’approche par compétences dans le cadre de la Nouvelle Gestion Publique. Dans J. Morrissette et M.-F. Legendre (dir.), Enseigner et évaluer : regards sur les enjeux éthiques et sociopolitiques (p. 211-245). Québec : Presses de l’Université Laval.

Litalien, R., Moore, D. et Sabatier, C. (2012). Ethnographie de la salle de classe, pratiques plurielles et réflexivité : pour une écologie de la diversité en contexte francophone en Colombie-Britannique. Revue canadienne de l'éducation, 35(2), 192-211.

Lorcerie, F. (2002). Éducation interculturelle : État des lieux. VEI Enjeux, 129, 170-189.

Lussier, D. (2007). Les compétences interculturelles : un référentiel en enseignement et en évaluation. Colloque ALTE/CIEP, Paris, France.

Ministère de l'Éducation de l'Ontario (MEO) (2009). Stratégie ontarienne d'équité et d'éducation inclusive. Toronto : Ministère de l'éducation. http://www.edu.gov.on.ca/fre/policyfunding/equity.pdf

Ministère de l’Éducation de la C.-B. (MECB) (2015b). Présentation des nouveaux programmes d'études de la Colombie-Britannique. Victoria : MECB. https://curriculum.gov.bc.ca/sites/curriculum.gov.bc.ca/files/pdf/curriculum_intro_fr.pdf

Ministère de l’Éducation de la Colombie-Britannique (MECB) (2001). Responsabilité sociale : Document-cadre. Victoria : MECB, Student Assessment and Program Evaluation Branch. https://www.bced.gov.bc.ca/perf_stands/f-rs-m-10.pdf

Ministère de l'Éducation de la Colombie-Britannique (MECB) (2004). Safe, caring and orderly schools: A Guide. Victoria : MECB. www.bced.gov.bc.ca/sco/guide/scoguide.pdf

Ministère de l'Éducation de la Colombie-Britannique (MECB) (2005). La diversité dans les écoles de la C.B. : un cadre de référence [révisé en 2008]. Victoria : MECB. http://www.bcedplan.ca/assets/pdf/bc_edu_plan_french.pdf

Ministère de l'Éducation de la Colombie-Britannique (MECB) (2008). Making space : Teaching for diversity and social justice throughout the K-12 curriculum. Victoria : MECB.

Ministère de l'Éducation de la Colombie-Britannique (MECB) (2013a). Exploring curriculum design. Victoria : MECB. http://www.bced.gov.bc.ca/irp/docs/exp_curr_design.pdf

Ministère de l'Éducation de la Colombie-Britannique (MECB) (2013b). Defining cross-cultural competencies. Transforming curriculum and assessment. Working draft. Victoria MECB. https://www.bced.gov.bc.ca/irp/docs/def_xcurr_comps.pdf. 
Ministère de l'Éducation de la Colombie-Britannique (MECB) (2015a). BC's Education Plan. Focus on Learning. Update, January 2015. Victoria : MECB. http://www.bcedplan.ca/assets/pdf/bcs_education_plan_2015.pdf

Ministère de l'Éducation de la Colombie-Britannique (MECB)(n.d.). Le Plan d'éducation de la Colombie-Britannique. Victoria : MECB. http://www.bcedplan.ca/assets/pdf/bc_edu_plan_french.pdf

Ministère de l'Éducation du Québec (MEQ) (2001). La formation à l'enseignement : les orientations, les compétences générales. Québec : MEQ.

Moodley, K. (1988). L'éducation multiculturelle au Canada : des espoirs aux réalités. Dans F. Ouellet (dir.), Pluralisme et école (p. 187-221). Québec : Institut québécois de recherche sur la culture.

Mucchielli, R. (2006). L'analyse de contenu des documents et des communications. Paris : ESF.

Perrenoud, P. (2004). Obligation de compétences et analyse du travail. Dans C. Lessard et P. Merrieu (dir.), L'obligation des résultats en éducation (p. 207-232). Québec : Presses de l’Université Laval.

Potvin, M. et Carr, P. (2008). La « valeur ajoutée » de l'éducation antiraciste : conceptualisation et mise en œuvre au Québec et en Ontario. Éducation et francophonie, 36(1), 197-216.

Potvin, M. et Larochelle-Audet, J. (2016). Les approches théoriques sur la diversité ethnoculturelle en éducation et les compétences essentielles du personnel scolaire. Dans M. Potvin, M.-O. Magnan et J. Larochelle-Audet (dir.), La diversité ethnoculturelle, religieuse et linguistique en éducation. Théorie et pratique (p. 110-127). Anjou : Fides.

Potvin, M., Magnan, M.-O. et Larochelle-Audet, J. (dir.) (2016). La diversité ethnoculturelle, religieuse et linguistique en éducation. Théorie et pratique. Anjou : Fides.

Ragoonaden, K. (2011). La compétence interculturelle et la formation initiale : le point sur le CECR et l'IDI. Revue canadienne de linguistique appliquée, 14(2), 86-105.

Ragoonaden, K. (2012). Le savoir interculturel : le Cadre européen commun de référence pour les langues (CECR), les indicateurs des savoirs et l'inventaire du développement interculturel (IDI). Journal de l'immersion, 34(1), 36-40.

Shariff, S. (2006). Balancing competing rights: a stakeholder model for democratic schools. Canadian Journal of Education, 29(2), 476-496.

Statistique Canada (2011). Un aperçu des statistiques sur les autochtones ( $2 \mathrm{e}$ éd) [en ligne]. http://www.statcan.gc.ca/pub/89-645-x/2015001/education-fra.htm

Sullivan, B. (1988). Royal Commission on Education. A legacy for learners : summary of findings. Province of British Columbia, Victoria. http://crofsblogs.typepad.com/files/legacyforlearnerssummary.pdf

Taylor, C. (1994). Multiculturalisme, différence et démocratie. Paris : Aubier.

Unesco (2009). L'Éducation pour l'inclusion : la voie de l'avenir. Rapport final de la Conférence internationale de l'éducation. $48^{\mathrm{e}}$ session, 25-28 novembre 2008. Genève : Unesco. 